\title{
Clinical research of percutaneous spinal nerve posteromedial ramus destruction in treatment for chronic facet arthrogenic low back pain: a randomized trial
}

\author{
Shuang Feng", Qiujin Xiao", Yinchu Shao, Yu Wu, Ting Yu, Wei Hu, Guoyun Bu, Hao Li \\ The 908th Hospital of Chinese PLA, Nanchang, China \\ Contributions: (I) Conception and design: S Feng, G Bu, H Li; (II) Administrative support: G Bu, H Li; (III) Provision of study materials or patients: \\ Q Xiao, Y Shao; Yu Wu; (IV) Collection and assembly of data: Y Wu, T Yu, W Hu; (V) Data analysis and interpretation: S Feng, G Bu, H Li; (VI) \\ Manuscript writing: All authors; (VII) Final approval of manuscript: All authors. \\ \#The authors contributed equally to this work. \\ Correspondence to: Hao Li. The 908th Hospital of Chinese PLA, Nanchang, China. Email: $47160843 @ q q . c o m$. Guoyun Bu. Department of \\ Orthopedics of Tianjin Hospital, Liberation Road 406, Hexi District Tianjin, China. Email: $517730611 @$ qq.com.
}

\begin{abstract}
Background: To investigate the clinical effect of percutaneous spinal nerve posteromedial ramus destruction in treatment for chronic facet arthrogenic low back pain.

Methods: A total of 118 patients with chronic facet arthrogenic low back pain were enrolled in our hospital from October 2015 to October 2018 and randomly divided into two groups. Of these patients, 59 received conservative treatment as the control group while 59 patients were treated with percutaneous spinal nerve posteromedial ramus destruction as the observation group. Pain score (Visual Analogue Scale, VAS), functional score (Oswestry Disability Index, ODI), treatment score (Japanese Orthopaedic Association, JOA), life quality score (Short Form 36-item Health Survey, SF-36), and lumbar mobility (range of motion, ROM) were evaluated before and after treatment. The treatment effect between two groups was compared.

Results: The VAS score and ODI score at 2, 4, and 12 weeks after treatment and last follow-up in the observation group were lower than those of the control group $(\mathrm{P}<0.05)$. JOA score, SF-36, and ROM were higher than those of the control group $(\mathrm{P}<0.05)$.

Conclusions: Percutaneous spinal nerve posteromedial ramus destruction has a significant effect in treatment for chronic facet arthrogenic low back pain, which can relieve pain, improve function, and enhance quality of life.
\end{abstract}

Keywords: Percutaneous spinal nerve posteromedial ramus destruction; chronic facet arthrogenic low back pain; quality of life; lumbar mobility

Submitted May 26, 2020. Accepted for publication Jul 13, 2020.

doi: 10.21037/apm-20-1374

View this article at: http://dx.doi.org/10.21037/apm-20-1374

\section{Introduction}

Lumbar facet joints, also called articular process joints, are synovial joints connected to the vertebrae of the lumbar. They are composed of the articular surfaces of the upper and lower articular processes of adjacent vertebrae, and are prone to internal degeneration of the joint, secondary to synovial hyperplasia, inflammatory changes, joint facial cartilage degeneration, and other diseases. These can cause chronic articular process joint-derived low back pain which seriously affects the patient's quality of life $(1,2)$, is a widespread concern in society, and a near-continuous focus of clinical research. Traditionally, physiotherapy has been more commonly used as a treatment but entails slow recovery and poor effectiveness, often resulting in relapse. There is also significant debate concerning the preferred surgical procedures and radiofrequency denervation for joint-derived low back pain $(3,4)$, and no unified standard has yet been established. Some studies suggested 


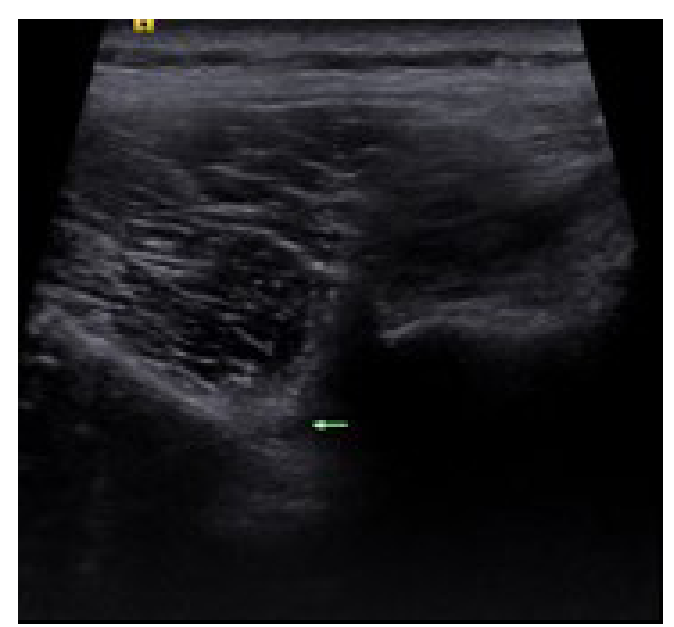

Figure 1 Localization of target area. The arrow shows the treatment target area, which is at the upper edge of the root of the transverse process, between the mastoid process and the accessory process.

that radiofrequency denervation resulted in significant reductions in low back pain originating from the facet joints in patients showing the best response to diagnostic block over the first 12 months when compared with sham procedures or epidural nerve blocks (4). In this study, we thus aimed to evaluate the effectiveness of percutaneous spinal nerve posterior medial branch lesions for patients with chronic articular process joint-derived low back pain. In this study, we calculated VAS, ODI, JOA, and SF-36 score to evaluate the effectiveness. We present the following article in accordance with the CONSORT reporting checklist (available at http://dx.doi.org/10.21037/apm-201374).

\section{Methods}

\section{Study population}

In our hospital, from October 2015 to October 2018, 118 patients with chronic articular facet joint-induced low back pain were enrolled according to inclusion and exclusion criteria. The inclusion criteria were as follows: (I) chronic low back pain for more than 3 months; (II) mechanical low back pain, with posture changes such as turning and twisting, and possibly accompanied by varying degrees of hip and lower limb involvement pain; (III) computed tomography (CT) scan showing facet hyperplasia, marginal osteophytes, joint vacuum sign, and calcification in or around the joint capsule. The exclusion criteria included patients with heart disease, renal failure, liver failure, mental diseases, contraindications to surgery, and infectious diseases. A random number table was used to divide patients into two groups: 59 cases were placed in the control group comprising 34 male cases and 25 female cases, aged 36-71 $(52.6 \pm 7.8)$ years old, with a duration of disease of $8-32$ (17.6 \pm 5.1$)$ months; 59 cases were placed in the observation group comprising 32 males and 27 females, aged $37-70$ $(52.1 \pm 8.9)$ years old, with a duration of disease of $9-31$ $(17.3 \pm 6.8)$ months. There were no statistically significant differences in the baseline characteristics of gender, age, or disease course between the two groups $(\mathrm{P}>0.05)$. All procedures performed in this study involving human participants were in accordance with the Declaration of Helsinki (as revised in 2013). This study was approved by the Ethics Committee of the 908th Hospital of Joint Logistics Support Force (Approval number 2015LL003) and informed consent was taken from all the patients.

\section{Study methods}

The control group was given conservative treatment in the form of deep muscle stimulation training; active and passive low back muscle training; oral celecoxib capsules (Pfizer Pharmaceutical Co., Ltd., Sinopharm J20120063) $200 \mathrm{mg} /$ time at 2 times/d; oral methylcobalamin tablets (North China Pharmaceutical Kangxin Co., Ltd., National Pharmaceutical Standard H20031126) $500 \mu \mathrm{g} / \mathrm{time}$, at 3 times/d, and a treatment course of 2 weeks.

The observation group implemented percutaneous spinal nerve posterior medial branch lesion treatment to help patients assume the prone position and create bending of the lumbar. After routine disinfection, the surgical target area was determined using ultrasound guidance. The location of the posterior branches of the L3 and L4 was the cross-point of the upper articular processes of L4 and L5 and the corresponding transverse processes; the posterior branch of the L5 was the focal point of the superior articular process of S1 and the sacral wing (Figure 1). Next, $0.5 \%$ lidocaine (Chenxin Pharmaceutical Co., Ltd.; National Pharmaceutical Standard, H20043560) was used for local anesthesia, with the needle tip perpendicular to the nerve to ensure no blood, cerebrospinal fluid, or air were drawn back; furthermore, a radio frequency electrode was connected and set to a frequency of $50 \mathrm{~Hz}$ with the voltage between 0.3 and $0.5 \mathrm{~V}$ to give accurate positioning and test sensory function. High-frequency stimulation in the painful 
Table 1 Comparison of VAS scores between two groups before and after treatment (points)

\begin{tabular}{lccccc}
\hline Group & Before treatment & 2 weeks & 4 weeks & 12 weeks & Last follow-up \\
\hline Control group $(\mathrm{n}=59)$ & $7.28 \pm 1.34$ & $2.76 \pm 0.58$ & $2.43 \pm 0.25$ & $2.38 \pm 0.19$ & $2.15 \pm 0.14$ \\
Observation group $(\mathrm{n}=59)$ & $7.31 \pm 1.21$ & $2.04 \pm 0.32$ & $1.87 \pm 0.14$ & $1.56 \pm 0.12$ & $1.32 \pm 0.10$ \\
$T$ value & 0.128 & 8.349 & 15.012 & 28.028 & 37.056 \\
P value & 0.898 & 0.000 & 0.000 & 0.000 & 0.000 \\
\hline
\end{tabular}

VAS, visual analog scale.

area was conducted to induce numbness and bloating, and subsequent exercise tests were performed. The set frequency was $2 \mathrm{~Hz}$ while the voltage was above $1 \mathrm{~V}$; low frequency stimulation was conducted to induce contraction of the lower extremity muscles without causing motor nerve stimulation. If the sensory test was successful and the exercise test did not induce lower extremity exercise, radiofrequency thermocoagulation damage could proceed. The parameters were set to $72{ }^{\circ} \mathrm{C}$ (Celsius degree) for $60 \mathrm{~s}$ and $80{ }^{\circ} \mathrm{C}$ for $90 \mathrm{~s}$. After the needle was withdrawn, the patient was instructed to lie down for 60 minutes. Patients were followed up for 6 months after operation.

Before treatment, 2, 4, and 12 weeks after treatment, and at last follow-up, the pain score (Visual Analogue Scale, VAS), function score (Oswestry Disability Index, ODI), treatment score (Japanese Orthopaedic Association Scores, JOA), quality of life score (Short Form 36-item Health Survey, SF-36), lumbar spine activity evaluation (range of motion, ROM), and the general effectiveness of the two groups (excellent, good, fair, poor) were all determined.

\section{Evaluation criteria}

For the pain scoring (5), A VAS scoring method was used, with a full score of 10 points. The higher the score, the more severe the pain. For function scoring (6), ODI was used, with a perfect score being 100 points. The higher the score, the more obvious the dysfunction. For treatment scoring (7), the JOA standard system was used, with a full score being 29 points. The higher the score, the better the lumbar function. For quality of life scoring (8), SF-36 quality of life index was used, with a perfect score of 100 points. The higher the score, the better the quality of life.

\section{Statistical methods}

SPSS19.0 software was used for statistical analysis. The measurement data are represented by mean \pm standard deviation $(\bar{x} \pm s)$ and determined by $t$ test, while the categorical variables are represented by rate (\%) and were analyzed be $\chi^{2}$ test. A P value less than 0.05 was considered statistically significant.

\section{Results}

\section{Comparison of VAS scores between the two groups before and after treatment}

The VAS score of the observation group was lower than that of the control group 2 weeks, 4 weeks, and 12 weeks after treatment. The difference was statistically significant $(\mathrm{P}<0.05)$ (Table 1).

\section{Comparison of ODI scores before and after treatment}

The ODI score in observation group at 2, 4, and 12 weeks after treatment and last follow-up was lower than that of the control group. The difference was statistically significant $(\mathrm{P}<0.05)$ (Table 2).

\section{Comparison of $\mathcal{O O A}$ score before and after treatment between the two groups}

The JOA score in observation group at 2 weeks, 4 weeks, and 12 weeks after treatment and last follow-up was higher than that of the control group. The difference was statistically significant $(\mathrm{P}<0.05)$ (Table 3).

\section{Comparison of SF-36 score before and after treatment}

The SF-36 score of the observation group was higher than that of the control group 2 weeks, 4 weeks, and 12 weeks after treatment and at last follow-up. The difference was statistically significant $(\mathrm{P}<0.05)($ Table 4). 
Table 2 Comparison of ODI scores between the two groups before and after treatment (points)

\begin{tabular}{lccccc}
\hline Group & Before treatment & 2 weeks & 4 weeks & 12 weeks & Last follow-up \\
\hline Control group $(\mathrm{n}=59)$ & $38.75 \pm 4.01$ & $18.50 \pm 1.74$ & $16.39 \pm 1.27$ & $15.46 \pm 1.35$ & $14.98 \pm 1.28$ \\
Observation group $(\mathrm{n}=59)$ & $38.69 \pm 3.64$ & $12.56 \pm 1.08$ & $11.92 \pm 0.95$ & $11.31 \pm 0.88$ & $11.04 \pm 0.76$ \\
$T$ value & 0.085 & 22.279 & 21.649 & 19.781 & 20.330 \\
$P$ value & 0.932 & 0.000 & 0.000 & 0.000 & 0.000 \\
\hline
\end{tabular}

ODI, Oswestry Disability Index.

Table 3 Comparison of JOA scores before and after treatment between the two groups (points)

\begin{tabular}{lccccc}
\hline Group & Before treatment & 2 weeks & 4 weeks & 12 weeks & Last follow-up \\
\hline Control group $(\mathrm{n}=59)$ & $13.49 \pm 1.58$ & $16.83 \pm 1.12$ & $17.95 \pm 1.30$ & $18.31 \pm 1.42$ & $18.95 \pm 1.13$ \\
Observation group $(\mathrm{n}=59)$ & $13.52 \pm 1.47$ & $20.16 \pm 1.39$ & $20.36 \pm 1.17$ & $21.87 \pm 1.25$ & $22.96 \pm 1.01$ \\
$T$ value & 0.109 & 14.329 & 10.584 & 14.454 & 20.323 \\
P value & 0.915 & 0.000 & 0.000 & 0.000 & 0.000 \\
\hline
\end{tabular}

JOA, Japanese Orthopaedic Association.

Table 4 Comparison of SF-36 scores before and after treatment between the two groups (points)

\begin{tabular}{lccccc}
\hline Group & Before treatment & 2 weeks & 4 weeks & 12 weeks & Last follow-up \\
\hline Control group $(\mathrm{n}=59)$ & $72.64 \pm 5.18$ & $80.34 \pm 2.85$ & $81.97 \pm 3.01$ & $82.04 \pm 3.12$ & $82.64 \pm 2.79$ \\
Observation group $(\mathrm{n}=59)$ & $72.53 \pm 4.62$ & $90.16 \pm 2.73$ & $90.85 \pm 3.49$ & $91.54 \pm 3.71$ & $92.28 \pm 2.65$ \\
$T$ value & 0.122 & 19.113 & 14.799 & 15.053 & 19.243 \\
$P$ value & 0.903 & 0.000 & 0.000 & 0.000 & 0.000 \\
\hline
\end{tabular}

SF-36, Short Form 36-item Health Survey.

\section{Comparison of ROM before and after treatment}

The ROM in the observation group was higher than that in the control group 2 weeks, 4 weeks, and 12 weeks after treatment and at last follow-up. The difference was statistically significant $(\mathrm{P}<0.05)$ (Table 5).

\section{Discussion}

Chronic facet joint-induced low back pain is a common clinical disease, which is common in middle-aged and elderly people, mainly due to the increased load caused by long-term poor posture (9-11). When the intervertebral disc degenerates, the height of the intervertebral disc will decrease, and the axial stress load will be partially transferred to the facet joint of the lumbar spine, resulting in an increase of load, which will cause secondary joint degeneration. The first affected area is the articular cartilage, which becomes susceptible to thinning, degeneration, and necrosis, leading to synovitis, with joint space narrowing and osteophyte hyperplasia. The joint capsule of the small joints is rich in nerves, including low-threshold mechanoreceptors, mechanosensitive pain receptors, and resting pain receptors. These receptors are exceptionally sensitive to the high tension and torsional stress, and thus have the tendency to generate pain. Inflammatory substances are the main stimulator to paingenerating nerve endings $(12,13)$. After degeneration and trauma, a large amount of inflammatory substances are released, which also increases pain and eventually induces chronic articular process arthritis-induced low back pain.

Transcutaneous posterior medial rachis nerve destruction is a surgical treatment that plays an important role in the diagnosis and treatment of chronic articular facet joint- 
Table 5 Comparison of ROM before and after treatment between the two groups $\left(^{\circ}\right)$

\begin{tabular}{lccccc}
\hline Group & Before treatment & 2 weeks & 4 weeks & 12 weeks & Last follow-up \\
\hline Control group $(\mathrm{n}=59)$ & $15.01 \pm 1.27$ & $17.28 \pm 1.36$ & $17.57 \pm 1.48$ & $17.89 \pm 2.01$ & $18.02 \pm 1.75$ \\
Observation group $(\mathrm{n}=59)$ & $14.95 \pm 1.06$ & $19.52 \pm 1.80$ & $19.84 \pm 1.73$ & $20.12 \pm 1.85$ \\
$T$ value & 0.279 & 7.627 & 7.659 & 6.270 & 7.316 \\
$P$ value & 0.781 & 0.000 & 0.000 & 0.000 & 0.000 \\
\hline
\end{tabular}

ROM, range of motion.

induced low back pain. The rationale for this treatment is as follows: the posterior medial spinal nerve branch is the only way for the lumbar facet joint to pass signal of pain up to central nervous system, and its physiological structure is relatively fixed; thus, the method of achieving local pain point injection block is feasible and can effectively block the pain signals. However, the use of drug injection therapy has drawbacks. When the drug effect disappears, the pain signal will continue, disturbing the efficacy of treatment. It therefore should not be the first choice. Through radiofrequency ablation technology, the posterior medial branch of the spinal nerve can be effectively cut off. Theoretically speaking, this can also completely cut off the path of the pain signal, and thus obtain significant longterm pain relief $(14,15)$. According to previous studies and our experience, the main problem with this technique is incomplete pain-killing in about $10 \%$ of these patients.

The results of this study showed that the VAS score and ODI score in the observation group were lower than those of the control group 2 weeks, 4 weeks, and 12 weeks after treatment and at the last follow-up. Meanwhile, the JOA score, SF-36 score, and ROM were higher than those of the control group. These results indicate that percutaneous posterior medial rachis destruction is an effective treatment for chronic articular facet joint-induced low back pain, which not only can reduce patients' pain and improve joint function, but can also significantly improve the quality of life of the patient.

However, treatment via posterior medial branch of the spinal nerve may also have some inherent flaws. The radiofrequency ablation range is a flat sphere with a radius of about $2 \mathrm{~mm}$. The ablation range directly in front of the electrode is much smaller than the ablation range on both sides, and the distal tissue of the electrode tip is difficult to damage. Thermal coagulation is also likely to cause incomplete damage to the target nerve coagulation, or incomplete blocking and inactivation, resulting in future relapse. In addition, percutaneous puncture is used for blind penetration, and it is impossible to observe whether the radiofrequency electrode is in contact with the nerve under direct vision. Only the C-arm X-ray machine can be used to adjust the electrode tip to the target position according to the bone sign. We use ultrasound-guided operation, which not only avoids the $\mathrm{X}$-ray radiation, but also enables the radiofrequency electrode to accurately locate the treatment target area and improve treatment effectiveness.

In summary, percutaneous spinal nerve posterior medial branch lesions are effective in treating chronic articular facet joint-induced low back pain and can reduce pain, improve function, and improve quality of life; thus, this treatment warrant furthers clinical application. However, some precaution should be taken when interpreting these findings, as the sample size of this study was small while the observation time was relatively short. It is necessary to increase the sample size, especially in multiple medical centers and extend the observation time to confirm the value of our research.

\section{Acknowledgments}

Funding: None.

\section{Footnote:}

Reporting Checklist: The authors have completed the CONSORT reporting checklist. Available at http://dx.doi. org/10.21037/apm-20-1374

Data Sharing Statement: Available at http://dx.doi. org/10.21037/apm-20-1374

Conflicts of Interest: All authors have completed the ICMJE 
uniform disclosure form (available at http://dx.doi. org/10.21037/apm-20-1374). The authors have no conflicts of interest to declare.

Ethical Statement: The authors are accountable for all aspects of the work in ensuring that questions related to the accuracy or integrity of any part of the work are appropriately investigated and resolved. All procedures performed in this study involving human participants were in accordance with the Declaration of Helsinki (as revised in 2013). This study was approved by the Ethics Committee of the 908th Hospital of Joint Logistics Support Force (Approval number 2015LL003) and informed consent was taken from all the patients.

Open Access Statement: This is an Open Access article distributed in accordance with the Creative Commons Attribution-NonCommercial-NoDerivs 4.0 International License (CC BY-NC-ND 4.0), which permits the noncommercial replication and distribution of the article with the strict proviso that no changes or edits are made and the original work is properly cited (including links to both the formal publication through the relevant DOI and the license). See: https://creativecommons.org/licenses/by-nc-nd/4.0/.

\section{References}

1. Meroni R, Piscitelli D, Ravasio C, et al. Evidence for managing chronic low back pain in primary care: a review of recommendations from high-quality clinical practice guidelines. Disabil Rehabil 2019. [Epub ahead of print].

2. Edmond SL, Werneke MW, Young M, et al. Directional preference, cognitive behavioural interventions, and outcomes among patients with chronic low back pain. Physiother Res Int 2019;24:e1773.

3. Zeitoun R, Balogh P, Amiri A, et al. Chronic low back pain. Skeletal Radiol 2020;49:805-7.

4. Lee CH, Chung CK, Kim CH. The efficacy of conventional radiofrequency denervation in patients with chronic low back pain originating from the facet joints:

Cite this article as: Feng S, Xiao Q, Shao Y, Wu Y, Yu T, $\mathrm{Hu}$ W, Bu G, Li H. Clinical research of percutaneous spinal nerve posteromedial ramus destruction in treatment for chronic facet arthrogenic low back pain: a randomized trial. Ann Palliat Med 2020;9(4):2187-2192. doi: 10.21037/apm-20-1374 a meta-analysis of randomized controlled trials. Spine J 2017;17:1770-80.

5. Teodorczyk-Injeyan JA, Triano JJ, Injeyan HS. Nonspecific Low Back Pain: Inflammatory Profiles of Patients With Acute and Chronic Pain. Clin J Pain 2019;35:818-25.

6. Verbrugghe J, Agten A, Stevens S, et al. Exercise Intensity Matters in Chronic Nonspecific Low Back Pain Rehabilitation. Med Sci Sports Exerc 2019;51:2434-42.

7. Kaito T, Matsuyama Y, Yamashita T, et al. Costeffectiveness analysis of the pharmacological management of chronic low back pain with four leading drugs. J Orthop Sci 2019;24:805-11.

8. Khalil JG, Smuck M, Koreckij T, et al. A prospective, randomized, multicenter study of intraosseous basivertebral nerve ablation for the treatment of chronic low back pain. Spine J 2019;19:1620-32.

9. Maher C, Underwood M, Buchbinder R. Non-specific low back pain. Lancet 2017;389:736-47.

10. Argyriou AA, Anastopoulou GG, Bruna J. Inconclusive evidence to support the use of minimally-invasive radiofrequency denervation against chronic low back pain. Ann Transl Med 2018;6:127.

11. Braun H, Geniez C, Degboe Y, et al. Prevalence of inflammatory posterior arch abnormalities on lumbar spine MRI in spondyloarthritis patients compared with low back pain patients. Eur Radiol 2019;29:6405-15.

12. Curtis L, Shah N, Padalia D. Facet Joint Disease. In: StatPearls. Treasure Island (FL): StatPearls Publishing; 2020.

13. Lim YZ, Wang Y, Cicuttini FM, et al. Association Between Inflammatory Biomarkers and Nonspecific Low Back Pain: A Systematic Review. Clin J Pain 2020;36:379-89.

14. Orhurhu V, Urits I, Grandhi R, et al. Systematic Review of Radiofrequency Ablation for Management of Knee Pain. Curr Pain Headache Rep 2019;23:55.

15. Boudier-Revéret M, Thu AC, Hsiao MY, et al. The Effectiveness of Pulsed Radiofrequency on Joint Pain: A Narrative Review. Pain Pract 2020;20:412-21.

(English Language Editor: J. Gray) 